Case Report

\title{
Reactivation of Tuberculosis Following Major Abdominal Surgery
}

\section{AbhishekA. Jha ${ }^{1}$, Rajesh Ballal ${ }^{2}$, Aditya G.K. ${ }^{3}$}

${ }^{1}$ Post Graduate, ${ }^{2}$ Professor and Unit Head, ${ }^{3}$ Assistant Professor, Department of General Surgery, J ustice K.S. Hegde M edical Academy, Mangalore.

*Corresponding Author : Abhishek A. Jha, Post Graduate, Department of General Surgery, Justice K S HEGDE Medical Academy, Mangalore.

Received

: 20.07.2016

Review Completed : 07.10.2016

Accepted

: 04.11 .2016

Keywords : Key words are TB: Tuberculosis ; LTBI: Latent Tuberculosis Infection; DOTS: Directly Observed Treatment Short Course ; AFB: Acid Fast Bacilli

\begin{tabular}{|c|}
\hline Access this article online \\
\hline Quick Response Code \\
\hline
\end{tabular}

\begin{abstract}
A 46 year old male presented with dysphagia to solids and vomiting. He was evaluated clinically, radiologically and by upper GI endoscopy. He was diagnosed to have carcinoma of the distal $1 / 3$ rd of the oesophagus extending onto the Fundus of stomach (Sievert III).A standard transhiatalesophagectomy and total gastrectomy with Roux-en-Y esophagesophageal reconstruction was done. His post op period was relatively uneventful and discharged on POD 22. He was readmitted for radiotherapy as per Protocol. (5040 cGY/25\#/ 5 weeks). He developed persistent cough with expectoration on day 5. A pulmonology consult was given and sputum for AFB wassent. He was tested positive for pulmonary tuberculosis.

This man had a significant past history of being treated for TB meningitis 20 years ago Treatment now was started as per DOTS regimen and was discharged after his course of radiotherapy. His Antitubercular drug therapy has been continued.

A search of the English literature survey revealed no similar articles of "Reactivation of Tuberculosis after abdominal surgery". The possible mechanism may be due to profound immunosuppression following malignancy and itstreatment.

It is worthwhile to consider Tuberculosis as a differential diagnosis in the appropriate clinical setting as a cause of unexplained fever.
\end{abstract}

\section{Introduction}

Mycobacterium tuberculosis may survive for decades in the human body in a state termed latent (2). Tuberculosis (TB) remains a leading infectious cause of mortality and morbidity worldwide.

As one-third of the world's population is estimated to be latently infected with M ycobacterium tuberculosis, people with latent tuberculosis infection (LTBI) represent a Significant reservoir for future disease reactivation. Previous studies have generated two alternative hypotheses about the modes for $M$. tuberculosis persistence in humans. One hypothesis is that during latency, M. tuberculosis enters a very slow replicating or non-replicating dormant state in Which the bacilli are insensitive to killing by the host immune system and antiTB drugs.

The other hypothesis is that during latency, M . tuberculosis replicates but is killed by the host immune system at a rate roughly equal to its replication rate. (2).

\section{Case History}

A 46 year old gentleman presented to surgery opd with complaints of dysphagia and vomiting since 6 months. Dysphagia was more for solids than to liquids. He had significant loss of apetite and loss of weight. There was no history of cough with expectoration. Patient had history of Tuberculous Meningitis diagnosed 20 years ago .Treated with anti-tubercular therapy for a period of 8 months. There was no significant per abdomen or respiratory system finding on examination. He was evaluated clinically and radiologically. An Upper G.I Endoscopy was done which showed a carcinoma of distal $1 / 3^{\text {rd }}$ of oesophagus extending into fundus of stomach (Sievert III). A Transhiatal esophagectomy and total gastrectomy with Roux-en-Y esophagojejunostomy was done. His post-operative period 
was grossly delayed and he went home only on Post Op day

22. This man was admitted for radiotherapy (5040 cGY/25\#/5 weeks). He developed cough with expectoration on day 5 . The pulmonologist who evaluated him asked for sputum AFB. This was tested positive for AFB staining. Treatment was started as per DOTS regimen and was discharged after 5 weeks of radio therapy. He is presently on Anti Tubercular therapy

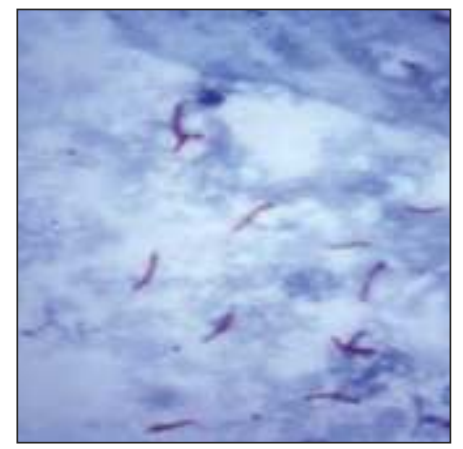

Figure 1 : Acid fast bacilli microscopy

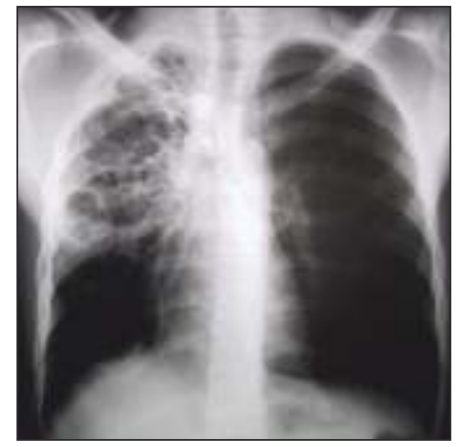

Figure 2 : Chest X ray during Radiotherapy day 5

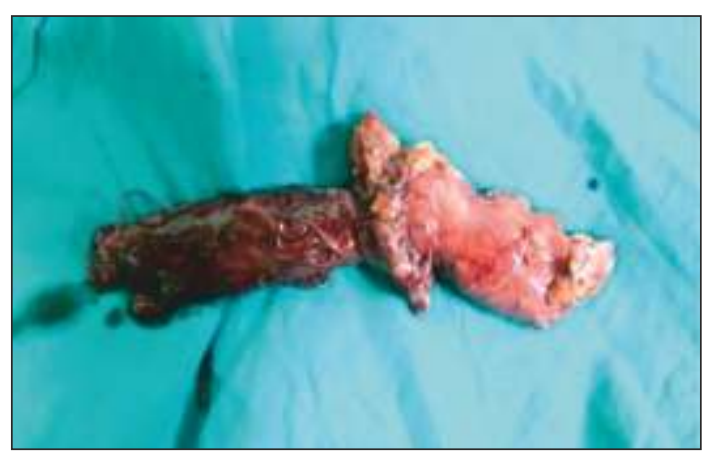

Figure 3 : Intra Op specimen of oesophagus for HPE

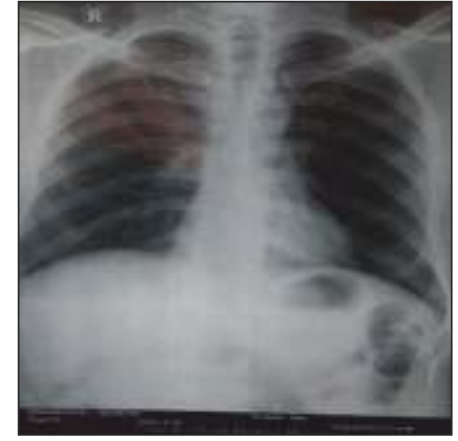

Figure 4 : Pre op Chest X Ray

\section{Discussion}

It is important to screen for reactivation of tuberculosis because it increases the mortality associated with malignancy. Neoplasms of the head and neck, gastrointestinal tract, lung and hematological have a high incidence of coexistence of Tuberculosis (1). Diagnosis of reactivated Tuberculosis may be delayed due to nonspecific symptoms. Cough and fever are common complaints among patients (1). The recommended treatment for TB comprises a 3-or 4-drug regimen. (1). A search of the english literature survey revealed no similar articles of "Reactivation of Tuberculosis after abdominal surgery". The possible mechanism may be due to profound immunosuppression following malignancy and its treatment. It is worthwhile to consider Tuberculosis as a differential diagnosis in the appropriate clinical setting as a cause of unexplained fever for malignancy.

\section{References}

1. Reactivation of tuberculosis in hepatocellular carcinoma treated with transcatheter arterial chemoembolization: A report of 3 cases. Yun Jeung Kim, Pyung Gohn Goh, Hee Seok M oon,et al2012 M ay 28; 4(5): 236-240 ISSN 1949-8470 (online)

2. How dormant is Mycobacterium tuberculosis during latency? A study integrating genomics andmolecular epidemiology Zhenhua Yanga, Mariana Rosenthala, Noah A.et al. Infect Genet Evol. 2011 Jul; 11(5):1164-7. doi: 10.1016/j.meegid.2011.02.002. Epub 2011 Feb 18. 